J. Clin. Chem. Clin. Biochem.

Vol. 19,1981 , pp. $55-60$

\title{
Selektive Charakterisierung von N1-Methyladenosin neben N7-Methylguanosin im Harn
}

Von E. Schlimme, K. S. Boos

Laboratorium für Biologische Chemie der Universität (GH) Paderborn und

M. Weise

Zentrum für Innere Medizin am Klinikum der Justus-Liebig-Universität Gießen

(Eingegangen am 30. Mai/29. September 1980)

Zusammenfassung: Die selektive Bestimmung der harngängigen, im Heterocyclus N-methylierten Ribonucleoside N1-Methyladenosin ( $\mathrm{m}^{1} \mathrm{Ado}$ ) und N7-Methylguanosin ( $\mathrm{m}^{7} \mathrm{Guo}$ ) neben anderen Nucleosiden wird beschrieben. Der Nachweis von $\mathrm{m}^{1}$ Ado und $\mathrm{m}^{7}$ Guo beruht auf chromatographischen Techniken unter Einbeziehung substanzspezifischer chemischer Reaktionen.

Die in Kinderurinen gemessenen $\mathrm{m}^{1}$ Ado-Werte zeigen neben einem offenbar altersabhängigen Gang eine deutliche Beziehung zur Höhe der Harnstoffausscheidung.

\section{Selective characterisation of N1-methyladenosine and N7-methylguanosine in urine}

Summary: We describe the selective analysis of $\mathrm{N}$-methylated ribonucleosides, i.e., N1-methyl adenosine ( $\mathrm{m}^{1} \mathrm{Ado}$ ) and N7-methyl guanosine $\left(\mathrm{m}^{7} \mathrm{Guo}\right)$ in the presence of other nucleosides in urine. $\mathrm{m}^{1}$ Ado as well as $\mathrm{m}^{7}$ Guo were characterized by use of chromatographic techniques including substance specific chemical reactions.

$\mathrm{m}^{1}$ Ado values in children urines seem to be age-dependent and related to urea elimination.

\section{Einführung}

Die analytische Erfassung der Grundbausteine von Nucleinsäuren in Körperflüssigkeiten eröffnet weitere diagnostischẻ Möglichkeiten im Bereich der klinischen Chemie $(1-6)$. Das Hauptinteresse der vorliegenden Arbeit gilt der selektiven Bestimmung der im Purinkern $\mathrm{N}$-methylierten Ribonucleoside N1-Methyladenosin ( $\mathrm{m}^{1} \mathrm{Ado}$ ) und N7-Methylguanosin $\left(\mathrm{m}^{7} \mathrm{Guo}\right)$ neben anderen Nucleosiden. N1-Methyladenosin wurde im T $\Psi$-loop (Position 58) vieler Transfer-Ribonucleinsäuren (tRNA) nachgewiesen (7). N7-Methylguanosin ist Bestandteil der 5'-terminalen Kappe eukaryotischer MessengerRibonucleinsäure (mRNA) $(8,9)$. Beide methylierten Nucleoside geben als Ausscheidungsprodukte im Urin somit Aufschluß über den Katabolismus ("turnover") von tRNA und mRNA und lassen damit Rückschlüsse auf den metabolischen Status, wie z.B. der Transkriptions- und Proteinbiosyntheserate, des Organismus zu.

\section{Material und Methoden}

\section{Nucleoside}

Adenosin (Ado), 2'-Desoxyadenosin (2' dAdo) und Guanosin (Guo) wurden von Boehringer, Mannheim, N1-Methyladenosin ( $m^{1}$ Ado), N6-Methyladenosin ( $m^{6}$ Ado), N7-Methylguanosin $\left(\mathrm{m}^{7} \mathrm{Guo}\right)$, Pseudouridin $(\Psi)$ und $2^{\prime}$-O-Methyladenosin (Ado ${ }^{\prime} \mathrm{m}$ ) von Sigma Chemical Company, St. Louis, Mo., USA, bezogen.

\section{Boronsäure-Biogel}

1 g Biogel Hydrazid (Bio-Gel P 2-Hydrazide, 200-400 mesh, Biorad Laboratories, Richmond, Cal., USA) wurde gemäß einer Literaturvorschrift (1) mit Bernsteinsäureanhydrid (Sigma, s. o.) umgesetzt; das succinylierte Gel wurde anschließend in Anwesenheit äquimolarer Mengen an 1-Ethyl-3-(3-dimethylaminopropyl)carbodiimid (EDAC) (Biorad, s.o.) mit $m$-Aminophenylboronsăurè (Sigma, s.o.) gekuppelt.

Unter alkalischen Bedingungen, d.h. nach Äquilibrierung des Boronsäure-Biogels in 0,25 mol/1 Ammoniumacetatpuffer, pH 8,8, ist das Affinitätsgel in der Lage, Nucleoside mit cisDiolgruppen selektiv zu binden $(1,2,10,11)$ während $2 . B$. 2'-Desoxyribonucleoside nicht retiniert werden. 


\section{Hochleistungsflüssigkeitschromatographie}

Die analytische Trennung der Nucleoside erfolgte mit Hilfe der Hochleistungsflüssigkeitschromatographie (HPLC) gemäß 1.c. (12) über eine Glassäule $(300 \times 5 \mathrm{~mm})$; stationäre Phase, Nucleosil 10 SA (Macherey und Nagel, Düren); mobile Phase, $0.4 \mathrm{~mol} / \mathrm{l}$ Ammoniumformiat $\mathrm{pH} \mathrm{4,1;} \mathrm{weitere} \mathrm{Angaben} \mathrm{siehe}$ Legende der Abbildungen 3 und 4.

\section{Vorbereitung des Harns}

$3 \mathrm{ml}$ Urin wurden tiefgefroren $\left(-20^{\circ} \mathrm{C}\right)$, aufgetaut, bei Raumtemperatur zentrifugiert und der Überstand abschließend durch ein Membranfilter (Millox $0.22 \mu \mathrm{m}$, Millipore, Buc, Frankreich) filtriert.

\section{Abtrennung der Ribonucleoside des Harns}

$3 \mathrm{ml}$ des keimfreien und partiell enteiweißten Harns wurden auf die mit Phenylboronsäure funktionalisierte Biogelsäule $(5 X$ $0,5 \mathrm{~cm}$ ) aufgegeben. Nach Äquilibrierung mit $0,25 \mathrm{~mol} / 1 \mathrm{Ammo}$ niumacetatpuffer, $\mathrm{pH} 8,8$, und Aufgabe des Harns wurde die Biogelsäule zunächst mit bidest. Wasser gewaschen. Hierdurch werden die ungeladenen Ribonucleoside wie z. B. Adenosin, Guanosin und Pseudouridin eluiert. Anschließend wurden mit $0,1 \mathrm{~mol} / 1$ Essigsäure die kationischen im Purinkern $\mathrm{N}$-methylierten Nucleoside wie $m^{1}$ Ado und $m^{7}$ Guo von der Biogelsäure abgelöst (Abb. 2).

\section{HPLC-Trennung der N-methylierten Ribonucleoside}

Das Eluat der im Kern N-methylierten Ribonucleoside (etwa $5-10 \mathrm{ml}$ ) wurde lyophilisiert und in $250 \mu \mathrm{l}$ bidest. Wasser gelöst. 5-10 $\mu$ l dieser Lösung wurden liquidchromatographisch analysiert (siehe Abb. 3, 4).

Ein Aliquot des Biogelsäule-Eluates wurde mit $\mathrm{NaOH}$ auf $\mathrm{pH} 10$ eingestellt, nach 4-5 h Reaktionszeit bei Raumtemperatur neutralisiert und davon wiederum $10 \mu$ liquidchromatographisch untersucht (siehe Abb. 3, 4). Die liquidchromatographische Differenzierungstechnik beruht auf dem chemischen Verhalten der $\mathrm{N}$-methylierten Ribonucleoside $\mathrm{m}^{1}$ Ado und $\mathrm{m}^{7}$ Guo. Unter alkalischen Bedingungen unterliegt $\mathrm{m}^{1}$ Ado einer Dimroth-Umlagerung unter irreversibler Bildung des stabilen N6-Methyladenosins, $\mathrm{m}^{6}$ Ado (Abb. 1).

Beim $\mathrm{m}^{7}$ Guo tritt im alkalischen Milieu (pH 9-10) eine Spaltung des Imidazolringes unter Bildung eines Pyrimidinnucleosides auf (Abb. 1) $(13,14)$, die ebenfalls beim Neutralisieren nicht reversibel ist.

Der Harnstoffgehalt wurde im enzymatischen Farbtest (TestCombination Boehringer, Mannheim) unter Einbeziehung einer internen Qualitätssicherung mit Precinorm ${ }^{R_{U}}$ (Boehringer, Mannheim) bestimmt.

\section{Ergebnisse und Diskussion}

Abbildung 2a zeigt das Elutionsdiagramm eines synthetischen Nucleosidgemisches in $0,25 \mathrm{~mol} / \mathrm{l}$ Ammoniumacetatpuffer, $\mathrm{pH} 8,8$, von einer Boronsäure-Biogelsäule.

In Abbildung $2 \mathrm{~b}$ ist das Elutionsdiagramm abgebildet, das bei der Chromatographie einer mit Ammoniumacetat $(0,25 \mathrm{~mol} / \mathrm{l})$ auf $\mathrm{pH} 8,8$ gepufferten und mit synthetischen Nucleosiden aufgestockten Urinprobe erhalten wurde.

Die „Recovery“ lag für die eingesetzten Nucleoside im untersuchten Konzentrationsbereich bei $\geqslant 90$ Prozent.

Abbildung $2 c$ schließlich zeigt als Beispiel ein Elutionsdiagramm einer reinen Harnprobe ( $3 \mathrm{ml}$ vorbereiteten Morgenurins s. o.; H.S. $\delta 11$ Jahre). Bei allen in der vorliegenden Arbeit untersuchten Harnproben (Tabelle 1)<smiles></smiles><smiles>CNc1ncnc2c1ncn2C1OC2OOC1C2O</smiles>

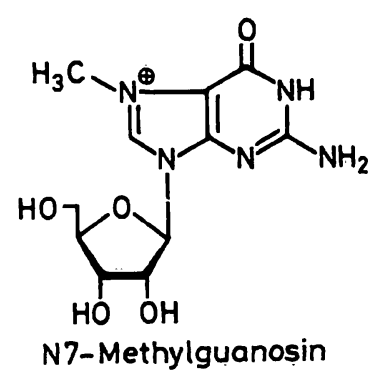

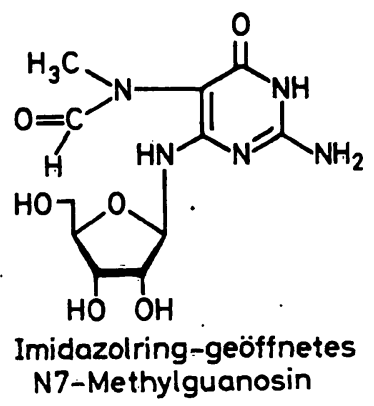

Abb. 1. Struktur von N1-Methyladenosin ( $\left.\mathrm{m}^{1} \mathrm{Ado}\right)$, N6-Methyladenosin ( $\left.\mathrm{m}^{6} \mathrm{Ado}\right)$, N7-Methylguanosin ( $\mathrm{m}^{7} \mathrm{Guo}$ ) und 2-Amino-4-ribofuranosylamido-5-formamidomethyl6-oxopyrimidin (Imidazolring-geöffnetem N7-Methylguanosin).

wurde - um die beschriebenen Umlagerungen von im Purinkern N-methylierten Ribonucleosiden weitgehend zu unterdrücken - eine Pufferung auf $\mathrm{pH} 8,8$ vermieden und der ursprüngliche $\mathrm{pH}$-Wert der Harnprobe unverändert belassen. Dieses war bei der Zielsetzung der Arbeit möglich, da eine selektive Bestimmung von $\mathrm{m}^{1}$ Ado neben $\mathrm{m}^{7} \mathrm{Guo}$ angestrebt wurde und deshalb auf eine Analyse aller wassereluierbaren Nucleoside verzichtet werden konnte.

Die Abbildungen 3 und 4 zeigen die HPLC-Chromatogramme der Nucleoside sowie als Beispiel eines über die Boronsäure-Biogelsäule gewonnenen nucleosidhaltigen Harneluates (H.S. o 11 Jahre; entspricht Elutionspeak III in Abb. 2c). Die Zuordnung der Retentionszeiten von $\mathrm{m}^{1}$ Ado und $\mathrm{m}^{6} \mathrm{Ado}$ bzw. $\mathrm{m}^{7}$ Guo und dem Imidazolringgeöffneten $\mathrm{m}^{7}$ Guo gelang mit authentischem Material unter Einbeziehung von UV-Spektren (Shimadzu UV-200 Spektrometer) für die Charakterisierung der Nucleoside $\mathrm{m}^{1}$ Ado $\left(\lambda_{\max }=258 \mathrm{~nm}, \epsilon=13,9 \mathrm{~cm}^{2} X\right.$ $\left.\mu \mathrm{mol}^{-1}, \mathrm{pH} 7\right), \mathrm{m}^{6}$ Ado $\left(\lambda_{\max }=266 \mathrm{~nm}, \epsilon=15,9 \mathrm{~cm}^{2}\right.$ $\left.X \mu \mathrm{mol}^{-1}, \mathrm{pH} 7\right), \mathrm{m}^{7} \mathrm{Guo}\left(\lambda_{\max }=259 \mathrm{~nm}\right.$ [Schulter bei $\left.\lambda=280 \mathrm{~nm}], \epsilon=9,7 \mathrm{~cm}^{2} \times \mu \mathrm{mol}^{-1}, \mathrm{pH} 7\right)$ und Imidazolring-geöffnetes $\mathrm{m}^{7} \mathrm{Guo}\left(\lambda_{\max }=265 \mathrm{~nm}, \epsilon=10 \mathrm{~cm}^{2} X\right.$ $\left.\mu \mathrm{mol}^{-1}, \mathrm{pH} 11\right)$.

Durch zusätzliche ${ }^{1} \mathrm{H}-\mathrm{NMR}$-spektroskopische Untersuchungen mit $m^{1}$ Ado und $m^{7}$ Guo konnte sichergestellt werden, dăß während der Vortrennung über die Boronsäure-Biogelsäure - wobei der Harn ohne Ammoniumacetatpufferzugabe (pH 8,8) auf die Säule aufgegeben und sofort mit Wasser eluiert wurde - nur etwa 5 Prozent des eingesetzten $m^{1}$ Ado in $m^{6}$ Ado umgelagert und $\leqslant 10$ Prozent $\mathrm{m}^{7}$ Guo in Imidazolring-geöffnetes $\mathrm{m}^{7} \mathrm{Guo}$ 

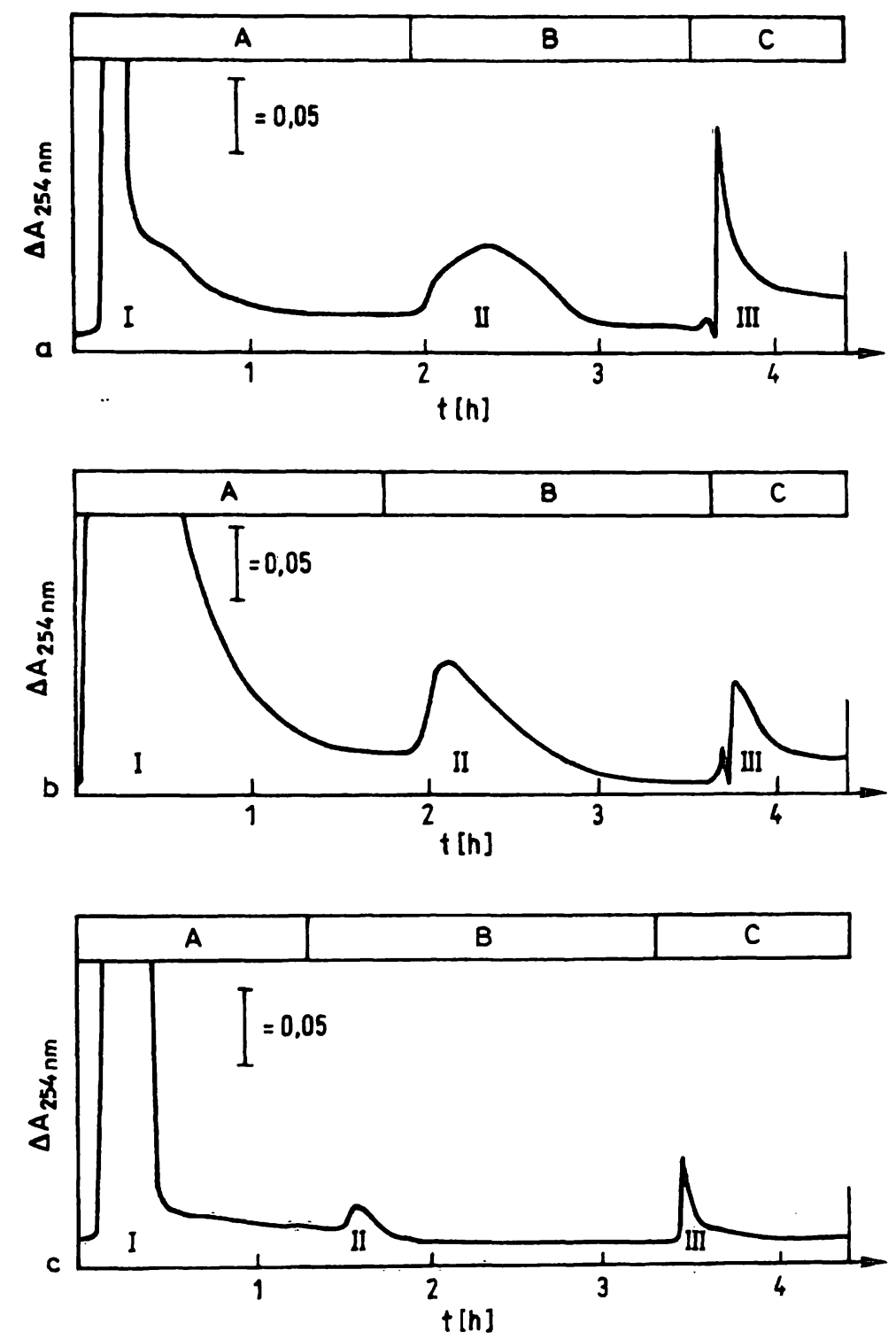

Abb. 2. Elutionsdiagramm von Boronsäure-Biogelsäule.

a) In $1 \mathrm{ml} \mathrm{0,25} \mathrm{mol} / 1$ Ammoniumacetatpuffer, pH 8,8 wurden $4 \mathrm{mmol}$ - in äquimolaren Konzentrationen - an Ado, $2^{\prime} \mathrm{dAdo}$, 2'-O-Methyl Ado, $\mathrm{m}^{1}$ Ado, Guo, $\mathrm{m}^{7} \mathrm{Guo}$ und $\Psi$ auf eine Boronsäure-Biogelsäule $(5 \times 0,5 \mathrm{~cm})$ gegeben und nacheinander mit (A) $0,25 \mathrm{~mol} / 1$ Ammoñiumacetạtpuffer, pH 8,8, (B) Wasser, (C) $0,1 \mathrm{~mol} / 1$ Essigsäure eluiert. Peak I: 2'dAdo, 2'-O-Methyl-Ado; Peak I (Schulter); $\Psi$; Peak II: Guo, Ado; Peak III: $\mathrm{m}^{1}$ Ado, $\mathrm{m}^{7}$ Guo.

b) $3 \mathrm{ml}$ eines vorbereiteten und auf $0,25 \mathrm{~mol} / 1$ Ammoniumacetat, $\mathrm{pH} 8,8$, eingestellten Urins (B. B. $\% 2,5 \mathrm{Jahre}$ ) wurden mit Nucleosiden bis auf 2'dAdo und 2'-O-Methyl Ado wie bei Abb. 2a beschrieben aufgestockt und über eine mit $0,25 \mathrm{~mol} / \mathrm{l}$ Ammoniumacetat, pH 8,8, äquilibrierte Boronsäure-Biogelsäule chromatographiert. Elutionsfolge: (A) $0,25 \mathrm{~mol} / 1$ Ammoniumacetatpuffer, pH 8,8, (B) Wasser, (C) 0,1 mol/1 Essigsäure. Peak I: $\Psi$ neben absorbierenden Harninhaltsstoffen; Peak II: Ado, Guo; Peak III: $\mathrm{m}^{1}$ Ado, $\mathrm{m}^{7}$ Guo.

c) $3 \mathrm{ml}$ vorbereiteter und auf $0,25 \mathrm{~mol} / 1$ Ammoniumacetat, $\mathrm{pH} 8,8$, eingestellter Urin (H. S. o $11 \mathrm{Jahre}$ ) wurde über eine mit $0,25 \mathrm{~mol} / 1$ Ammoniumacetatpuffer, p.H 8,8, äquilibrierte Boronsäure-Biogelsäule chromatographiert. Die Elution erfolgte wie in der Legende (Abb. 2b) beschrieben. Peak I: $\Psi$ und Desoxyribonucleoside neben absorbierenden Harninhaltsstoffen; Peak II: Ribonucleoside (nicht näher charakterisiert); Peak III: charakterisiert wurde Hauptkomponente $m^{1}$ Ado neben m $^{7}$ Guo.

aufgespalten wurden. Bei einer Vortrennung mit $0,25 \mathrm{~mol} / 1$ Ammoniumacetatpuffer, $\overline{\mathrm{pH}} 8,8$, über etwa 2 Stunden (Abb. 2c) ist mit einer $\mathrm{m}^{1} \mathrm{Ado} \rightarrow \mathrm{m}^{6}$ Ado Umlagerung von etwa 10 Prożent und mit einer $\mathrm{m}^{7}$ GuoSpaltung von etwa 15 Prozent zu rechnen.

In den bisher untersuchten Urinen (siehe Tab. 1) konnte $\mathrm{m}^{1}$ Ado als Hauptkomponente der $\mathrm{N}$-methylierten Nucleoside nachgewiesen werden. Dieser Befund bestä- tigt frühere Beobachtungen $(1,15)$, nach denen $\mathrm{m}^{1}$ Ado ausgeschieden wird und nachgewiesenes $m^{6}$ Ado ein während der Analyse durch Umlagerung aus $\mathrm{m}^{1}$ Ado entstandenes artefizielles Produkt ist. Der Ausscheidungsspiegel an $\mathrm{m}^{7}$ Guo ist um eine Größenordnung geringer. Der Nachweis von $\mathrm{m}^{1} \mathrm{Ado}$ im Bereich von $5-10 \mathrm{mg} \times \mathrm{1}^{-1}$ im Urin des Menschen wirft die Frage auf, ob diese Verbindung als Modell für die renale Elimination von Ri- 


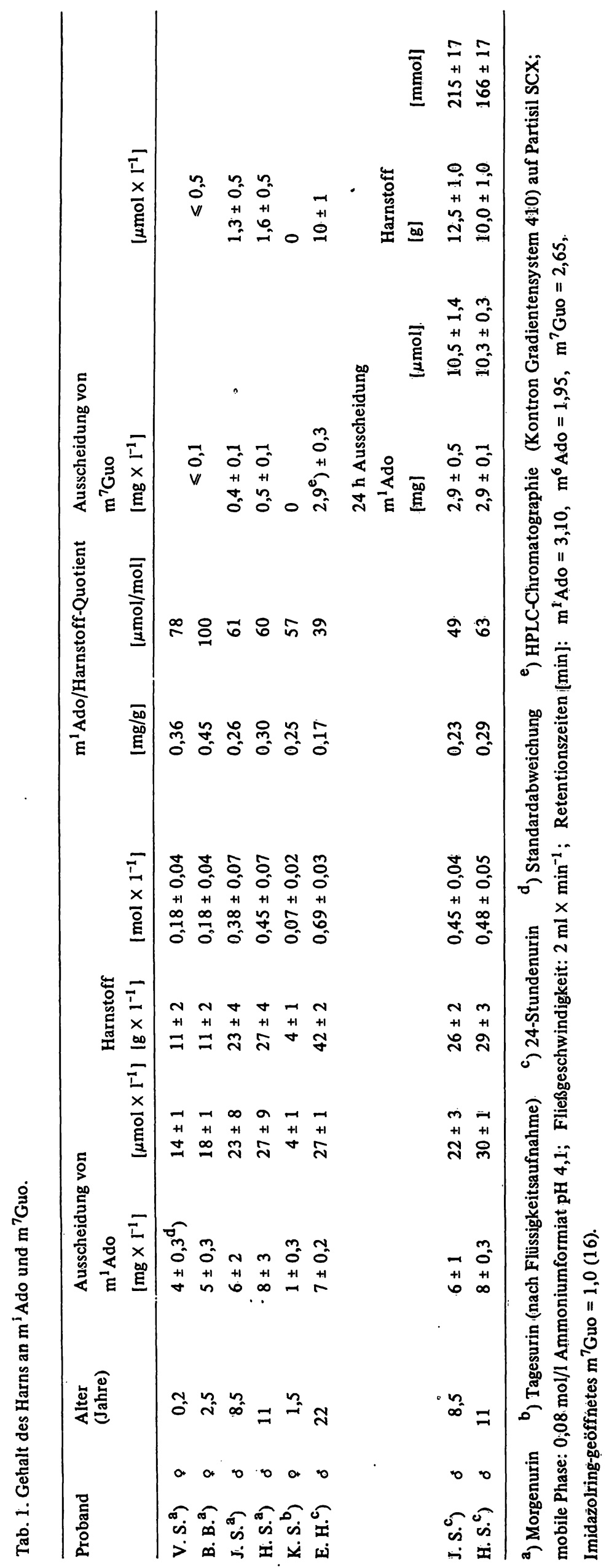

J. Clin. Chem. Clin. Biochem. / Vol. 19, 1981 / No. 2 


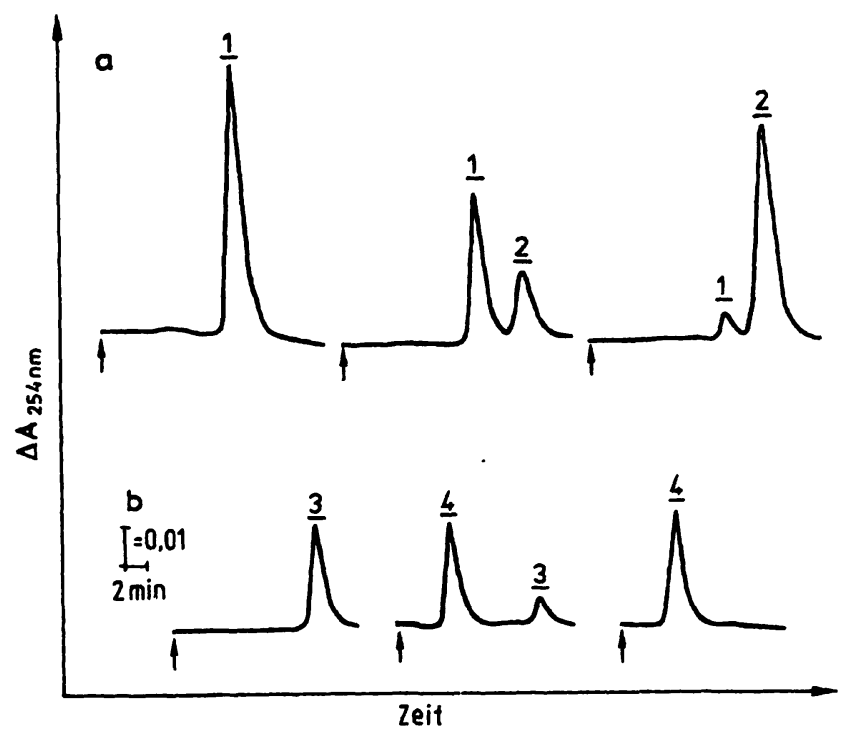

Abb. 3. HPLC-Analyse von $m^{1}$ Ado und $m^{7}$ Guo

a) HPLC-Diagramme vor, während und nach der Umlagerung (in dieser Reihenfolge) von $m^{1} A d o(1)$ in $\mathrm{m}^{6}$ Ado (2). Retentionszeiten $\mathrm{R}_{\mathrm{t}}[\mathrm{min}]: \mathrm{m}^{1}$ Ado $(1)=$ 14,$0 ; m^{6}$ Ado $(2)=18,8$.

b) HPLC-Diagramme vor, während und nach der Umlagerung (in dieser Reihenfolge) von $m^{7}$ Guo (3) in das ringoffene Pyrimidinderivat (4). Retentionszeiten $R_{t}$ [min]: $m^{7}$ Guo $(3)=14,8 ;(4)=5,6$.

Fließgeschwindigkeit der mobilen Phase: $18 \mathrm{ml} \times \mathrm{h}^{-1}$ bei einem Druck von 800 pounds/inch ${ }^{2}$.

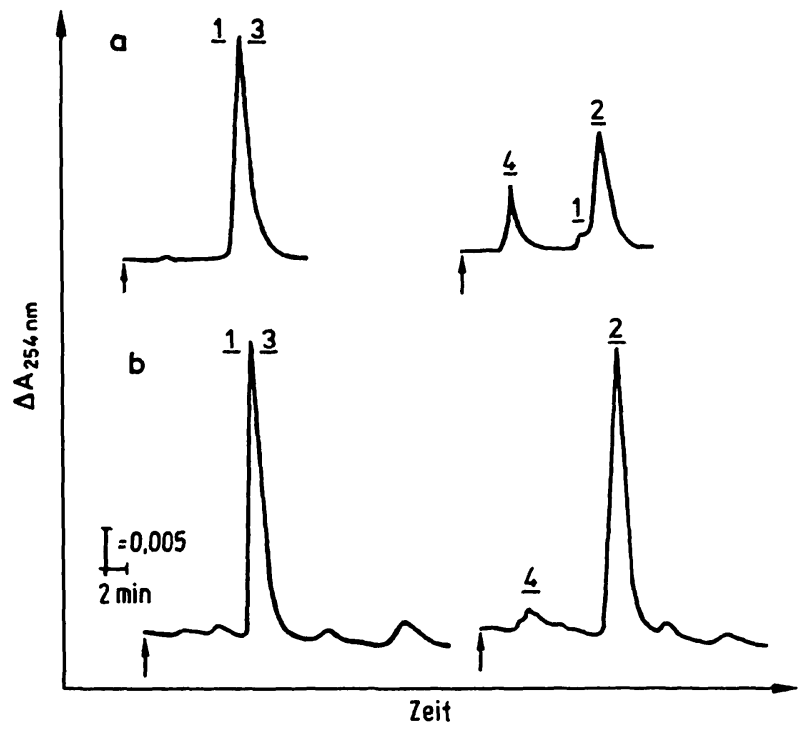

Abb. 4. HPLC-Analyse von $m^{1}$ Ado neben $m^{7}$ Guo

a) HPLC-Diagramme eines Gemisches von $m^{1}$ Ado (1) und $m^{7} G u o(3)$ vor und nach der Umlagerung in $\mathrm{m}^{6}$ Ado (2) und ringoffenes Pyrimidinderivat (4). Retentionszeiten $R_{t}[\mathrm{~min}]: \mathrm{m}^{1}$ Ado (1), $\mathrm{m}^{7} \mathrm{Guo}(3)=$ 12,$8 ;(4)=5,4, \mathrm{~m}^{6}$ Ado $(2)=14,8$.

b) HPLC-Diagramme eines Boronsäure-Biogel-Eluates (Aliquot von Peak III, Abb. $2 c$ - entspricht etwa $120 \mu$ l Ausgangsharn -, H. S. o 11 Jahre) vor und nach der Umlagerung unter alkalischen Bedingungen. Retentionszeiten $R_{t}[\min ]: m^{1}$ Ado (1), $m^{7}$ Guo (3) 12,$8 ;(4)=5,4, m^{6}$ Ado $(2)=14,8$.

Fließgeschwindigkeit der mobilen Phase: $18,5 \mathrm{ml}^{\circ} \mathrm{h}^{-1}$ bei einem Druck von 900 pounds/inch ${ }^{2}$.

suchten Kinderurinen liegt bei $67(\mathrm{~s}= \pm 17) \mu \mathrm{m} \mathrm{l} / \mathrm{mol}$. Die Einbeziehung von Urin mit pathobiochemischen Ausscheidungsmustern wird zeigen, ob der $\mathrm{m}^{1} \mathrm{Ado} / \mathrm{Harn}$ stoff-Quotient eine klinisch-diagnostisch verwertbare Aussage erlaubt.

\section{Danksagung}

Wir danken Frau Renate Schroer für geschickte technische Mitarbeit. Die Arbeit wurde von der Deutschen Forschungsgemeinschaft finanziell gefördert.

\section{Literatur}

1. Uziel, M., Smith, L. H. \& Taylor, S. A. (1976), Clin. Chem. $22,1451-1455$

2. Davis, G. E. Suits, R. D.;Kuo, K. C., Gehrke, C. W., Waalkes, T. P. \& Borek, E. (1977), Clin. Chem. 23, 1427-1435.

3. Solle, M. (1977), J. Clin. Chem. Clin. Biochem. 15, 371-374.

4a. Schöch, G., Winkler, K., Heller-Schöch, G. \& Baisch, H. (1979), Klin. Pädiatr. 191, 197-204.

b. Schöch, G., Heller-Schöch, G., Lorenz, H., Baisch, H., Weser, M., Leifer, W. \& Grüttner, R. (1977), Mschr. Kinderheilk. $125,542-543$. 5a. Chheda, G. B., Hong, C. I., Dulta, S. P., De, N. C. \& Parthasavathy, R. (1974), in Proc. of the Symp. of the Recent Developments in Oligonucleotide Synthesis and the Chemistry of Minor Bases of tRNA, Polish Academy of Sciences, Poznan, Polen, p. 3-33.

b. De, N. C. \& Chheda, G. B. (1979), J. Carbohydrates, Nucleosides, Nucleotides $6,371-385$.

6. Park, R. W., Holland, J. F. \& Jenkins, A. (1962), Cancer Res. $22,469-477$. 
7. Sprinzl, M., Grüter, F., Spelzhaus, A. \& Gauss, D. H. (1980), Nucleic Acid Research (Special Supplement) 8, r1-r22.

8. Shatkin, A. J. (1976), Cell 6, 645-653.

9. Filipowicz, W. (1978), FEBS-Lett. 96, 1-11.

10. Conner, J. M. \& Bulgrin, V. C. (1967), J. Inorg. Nucl. Chem. $29,1953-1961$.

11. Schott, H. (1972), Angew. Chem. 84, 819-820.

12a. Stahl, K. W., Schlimme, E. \& Schäfer, G. (1973), J. Chromatogr. 76, 477-481.

b. Stahl, K. W., Schlimme, E. \& Bojanovski, D. (1974), J. Chromatogr. 83, 395-404. 13a. Brookes, P. \& Lawley, P. D. (1960), J. Chem. Soc. 539-545. b. Brookes, P. \& Lawley, P. D. (1961), J. Chem. Soc. 39233928.

14. Haines, J. A., Reese, C. B. \& Lörd Todd, A. (1962), J. Chem. Soc. $5281-5288$.

15. Fink, K. \& Adams, W. S. (1968), Arch. Biochem. Biophys. $126,27-33$.

16. Schlimme, E., Hagemeier, E., Boos, K. S. \& Weise, M. (1980), j. Clin. Chem. Clin. Biochem. 18, 696-697 (Abstract).

Prof. Dr. Dr. Eckhard Schlimme Laboratorium für Biologische Chemie der Universität (GH)

Warburger Straße 100

D-4790 Paderborn 